Bull. Korean Math. Soc. 51 (2014), No. 5, pp. 1291-1297

http://dx.doi.org/10.4134/BKMS.2014.51.5.1291

\title{
VALUE DISTRIBUTION OF DIFFERENCE OPERATOR ON MEROMORPHIC FUNCTIONS
}

\author{
Jie Ding And Lian-Zhong Yang
}

\begin{abstract}
In this paper, we investigate the value distribution of the difference operator on meromorphic functions, and obtain a difference analogue of a theorem of Hayman on meromorphic functions.
\end{abstract}

\section{Introduction}

A function $f(z)$ is called meromorphic, if it is analytic in the complex plane except at isolated poles. It is assumed that the reader is familiar with the standard symbols and fundamental results of Nevanlinna theory such as the characteristic function $T(r, f)$, proximity function $m(r, f)$, counting function $N(r, f)$, the first and second main theorem etc., (see [11], [15], [23]). In addition we use $\sigma(f)$ to denote the order of growth of the meromorphic $f(z)$ and $\lambda(f)$ to denote the exponent of convergence of the zeros of $f(z)$. The notation $S(r, f)$ denotes any quantity that satisfies the condition: $S(r, f)=o(T(r, f))$ as $r \rightarrow \infty$ possibly outside an exceptional set of $r$ of finite logarithmic measure. Let $a(z)$ be a meromorphic function, we say that $a(z)$ is a small function of $f(z)$, if $T(r, a)=S(r, f)$.

In 1959, Hayman proved the following theorems.

Theorem A (see [10], Theorem 8). Let $f$ be a nonconstant transcendental entire function, let $n \geq 3$ be an integer, and a be a non-zero constant. Then $f^{\prime}(z)-a f^{n}(z)$ assumes all finite values infinitely often.

Theorem B (see [10], Theorem 9). Let $f$ be a nonconstant transcendental meromorphic function, let $n \geq 5$ be an integer, and a be a non-zero constant. Then $f^{\prime}(z)-a f^{n}(z)$ assumes all finite values infinitely often.

Recently, there have been significant results on Nevanlinna theory with respect to difference operators, see, the papers $[7,8]$ by Halburd and Korhonen;

Received February 3, 2012; Revised September 25, 2012.

2010 Mathematics Subject Classification. 30D35, 39A05.

Key words and phrases. meromorphic function, difference, shift, finite order.

This work is supported by the Research Project Supported by Shanxi Scholarship Council of China (No. 2013-045) and the Foundation Research Project of Shanxi Province (No. 2014021009-3). 
[5] by Chiang and Feng. Many papers (see [2], [4], [9], [11-20]) have focused on complex differences and given many difference analogues in value distribution theory of entire functions.

Liu and Laine [18] obtain the following theorem.

Theorem C (see [18], Theorem 1.1). Let $f$ be a transcendental entire function of finite order, not of period $c$, where $c$ is a non-zero constant, and let $s(z)$ be a nonzero small function of $f$. Then the difference polynomial $f^{n}(z)+f(z+$ c) $-f(z)-s(z)$ has infinitely many zeros in the complex plane, provided that $n \geq 3$.

In [3], Chen considered the difference counterpart of Theorem A and proved a difference analogue of Hayman's Theorem on entire function.

Theorem D (see [3], Theorem 1.1). Let $f$ be a transcendental entire function of finite order, not of period $c$, and let $a(\neq 0), b, c(\neq 0)$ be three complex numbers. Then $\Psi_{n}(z)=f(z+c)-f(z)-a f^{n}(z)$ assumes all finite values infinitely often, provided that $n \geq 3$, and for every $b$ one has $\lambda\left(\Psi_{n}(z)-b\right)=\sigma(f)$.

Qi and Liu [22] investigated the existence of transcendental entire solutions of non-linear difference equations. As an application, they obtained the following result.

Theorem E (see [22], Theorem 2). Let $f$ be a transcendental entire function of finite order, $c$ be a non-zero constant, $m$ and $n$ be integers satisfying $n \geq m>0$, and let $\lambda, \mu$ be two complex numbers such that $|\lambda|+|\mu| \neq 0$. If $n \geq 2$, then either $f^{n}(z)\left(\lambda f^{m}(z+c)+\mu f^{m}(z)\right)$ assumes every non-zero finite values infinitely often or $f(z)=\exp \left\{\frac{\log t}{c} z\right\} g(z)$, where $t=\left(-\frac{\mu}{\lambda}\right)^{\frac{1}{m}}$, and $g(z)$ is a periodic function with period $c$.

The purpose of this paper is to study value distribution of meromorphic function with respect to differences. Our methods of proof are also different from those in the previous theorems.

Theorem 1.1. Let $f$ be a non-constant meromorphic function of finite order, $s(z)$ be a small function of $f(z)$. Suppose that $P(z)$ is a polynomial, $m$ is the cardinality of the set $\{z: P(z)=0\}$ and $\operatorname{deg}(P(z))-m>3$, then $P(f(z))+$ $f(z+c)-s(z)$ has at least one zero. If $f$ is a transcendental meromorphic function, then $P(f(z))+f(z+c)-s(z)$ has infinitely many zeros.

Zhang and Korhonen [24] researched the value distribution of $q$-difference of meromorphic function. Corresponding to Theorem 1.1, we give an analogue result in $q$-differences as follows.

Theorem 1.2. Let $f$ be a meromorphic function of zero order, $q \in \mathbb{C} \backslash\{0\}, s(z)$ and $P(z)$ satisfy the condition of Theorem 1.1. Then $P(f(z))+f(q z)-s(z)$ has at least one zero. If $f$ is a transcendental meromorphic function, then $P(f(z))+f(q z)-s(z)$ has infinitely many zeros.

Remark 1. Some ideas of this paper are based on [6]. 


\section{Some lemmas}

In order to prove our theorem, we need the following lemmas:

Lemma 2.1 is a difference analogue of the logarithmic derivative lemma, given by Halburd and Korhonen [8] and Chiang and Feng [5] independently.

Lemma 2.1 (see [8], Theorem 2.1). Let $f(z)$ be a meromorphic function of finite order, and let $c \in \mathbb{C}$ and $\delta \in(0,1)$. Then

$$
m\left(r, \frac{f(z+c)}{f(z)}\right)+m\left(r, \frac{f(z)}{f(z+c)}\right)=O\left(\frac{T(r, f)}{r^{\delta}}\right)=S(r, f)
$$

for all $r$ outside of a possibly exceptional set with finite logarithmic measure.

Lemma 2.2 (see [23], Theorem 1.12). Let $f(z)$ be a non-constant meromorphic function, and let $P(f)=a_{0} f^{n}+a_{1} f^{n-1}+\cdots+a_{n}$, where $a_{0}(\neq 0), a_{1}, \ldots, a_{n}$ are small functions of $f$. Then

$$
T(r, P(f))=n T(r, f)+S(r, f) .
$$

Lemma 2.3 (see [1], Theorem 1.1). Let $f$ be a non-constant zero-order meromorphic function, and $q \in \mathbb{C} \backslash\{0\}$. Then

$$
m\left(r, \frac{f(q z)}{f(z)}\right)=S(r, f(z)) .
$$

Lemma 2.4 (see [24], Theorem 1.3). Let $f$ be a non-constant zero-order meromorphic function, and $q \in \mathbb{C} \backslash\{0\}$. Then

$$
N(r, f(q z))=N(r, f(z))+S(r, f(z))
$$

on a set of lower logarithmic density 1.

Lemma 2.5. Let $f(z)$ be a meromorphic function of finite order, $c \in \mathbb{C}$. Then

$$
N(r, f(z+c))=N(r, f(z))+S(r, f(z)) .
$$

Proof. Using Lemma 2.1 and the formula (12) in [13]

$$
N(r, f(z+c)) \leq N(r+|c|, f)=N(r, f(z))+S(r, f(z)) .
$$

Replacing $f(z)$ with $f(z-c)$, we have

$$
N(r, f(z)) \leq N(r, f(z-c))+S(r, f(z-c))=N(r, f(z-c))+S(r, f(z))
$$

for every $c \in \mathbb{C}$, so we deduce that

$$
N(r, f(z)) \leq N(r, f(z+c))+S(r, f(z)) .
$$

From (2.1) and (2.2), we obtain that

$$
N(r, f(z+c))=N(r, f(z))+S(r, f(z)) .
$$

Thus we completed the proof. 


\section{Proof of theorems}

Proof of Theorem 1.1. We define $\Phi(z)=P(f)+f(z+c)-s(z)$. Obviously, $\Phi(z) \not \equiv C$. If it is false, then $P(f) \equiv f(z+c)-s(z)+C$, so we have that

$$
T(r, P(f))=n T(r, f)+S(r, f)=T(r, f(z+c))+S(r, f),
$$

where $n=\operatorname{deg}(P)>3$. Using Lemma 2.1 and Lemma 2.5, we deduce that

$$
\begin{aligned}
T(r, f(z+c)) & =m(r, f(z+c))+N(r, f(z+c)) \\
& \leq m(r, f(z))+m\left(r, \frac{f(z+c)}{f(z)}\right)+N(r, f(z))+S(r, f(z)) \\
& =T(r, f(z))+S(r, f(z)) .
\end{aligned}
$$

The (3.1) and (3.2) imply $T(r, f(z))=S(r, f(z))$, a contradiction, Therefore $\Phi(z) \not \equiv C$.

Furthermore, we claim that

$$
\frac{P^{\prime}(f) f^{\prime}}{P(f)}-\frac{\Phi^{\prime}}{\Phi} \not \equiv 0
$$

Suppose contrary to the claim that $\frac{P^{\prime}(f) f^{\prime}}{P(f)}-\frac{\Phi^{\prime}}{\Phi} \equiv 0$. By integration we obtain $\Phi(z)=a P(f(z))$, where $a$ is a constant, hence $(a-1) P(f(z))=f(z+$ $c)-s(z)$.

If $a=1$, we can deduce $T(r, f(z+c))=T(r, s(z))$. This contradicts with the hypothesis.

Let $a \neq 1$. By the similar argument as the Case of $\Phi(z) \equiv C$, we get a contradiction. So the claim is true.

By a simple calculation we get that

$$
P(f)=\frac{\frac{\Phi^{\prime}}{\Phi}[f(z+c)-s(z)]-[f(z+c)-s(z)]^{\prime}}{\frac{P^{\prime}(f) f^{\prime}(z)}{P(f)}-\frac{\Phi^{\prime}}{\Phi}} .
$$

From Lemma 2.1, Lemma 2.2 and the First Fundamental Theorem, we obtain that

$$
\begin{aligned}
& T(r, P(f))=n T(r, f(z))+S(r, f) \\
= & T\left(r, \frac{\frac{\Phi^{\prime}}{\Phi}[f(z+c)-s(z)]-[f(z+c)-s(z)]^{\prime}}{\frac{P^{\prime}(f) f^{\prime}(z)}{P(f)}-\frac{\Phi^{\prime}}{\Phi}}\right) \\
\leq & m(r, f(z))+N\left(r, \frac{\Phi^{\prime}}{\Phi}[f(z+c)-s(z)]-[f(z+c)-s(z)]^{\prime}\right) \\
& +m\left(r, \frac{\Phi^{\prime}}{\Phi}-\frac{[f(z+c)-s(z)]^{\prime}}{[f(z+c)-s(z)]}\right)+m\left(r, \frac{P^{\prime}(f) f^{\prime}(z)}{P(f)}-\frac{\Phi^{\prime}}{\Phi}\right)
\end{aligned}
$$




$$
+N\left(r, \frac{P^{\prime}(f) f^{\prime}(z)}{P(f)}-\frac{\Phi^{\prime}}{\Phi}\right)+S(r, f(z)) .
$$

In the following we will estimate $N\left(r, \frac{\Phi^{\prime}}{\Phi}[f(z+c)-s(z)]-[f(z+c)-s(z)]^{\prime}\right)$ and

$$
N\left(r, \frac{P^{\prime}(f) f^{\prime}(z)}{P(f)}-\frac{\Phi^{\prime}}{\Phi}\right) .
$$

The poles of $\varphi_{1}(z)=\frac{\Phi^{\prime}}{\Phi}[f(z+c)-s(z)]-[f(z+c)-s(z)]^{\prime}$ come from the zeros of $\Phi(z)$, the poles of $P(f)$, the poles of $f(z+c)$ and the poles of $s(z)$. By hypothesis, $N(r, s)=S(r, f)$. In the following we always assume that $z_{0}$ is not a pole of $s$, let $z_{0}$ be a zero of $\Phi(z)$ or a pole of $P(f)$ but not a pole of $f(z+c)$, then $z_{0}$ is a simple pole of $\varphi_{1}(z)$; if $z_{0}$ is a common pole of $P(f)$ and $f(z+c)$, and the multiplicity is $m$ and $n$, respectively, then $z_{0}$ is a pole of $\varphi_{1}(z)$ with the multiplicity no more than $n+1$; if $z_{0}$ is a pole of $f(z+c)$ but not a pole of $P(f)$, we obtain that $z_{0}$ is a simple pole of $\varphi_{1}(z)$ because of (3.3). Using Lemma 2.5, we can get that

$$
\begin{aligned}
& N\left(r, \frac{\Phi^{\prime}}{\Phi}[f(z+c)-s(z)]-[f(z+c)-s(z)]^{\prime}\right) \\
\leq & \bar{N}\left(r, \frac{1}{\Phi(z)}\right)+\bar{N}(r, P(f))+N(r, f(z+c))+S(r, f(z)) \\
= & \bar{N}\left(r, \frac{1}{\Phi(z)}\right)+\bar{N}(r, f(z))+N(r, f(z))+S(r, f(z)) .
\end{aligned}
$$

We deal with the poles of $s(z)$ as above. The zeros of $\Phi(z)$, the poles of $P(f(z))$, the poles of $f(z+c)$ and the zeros of $P(f(z))$ compose the poles of $\varphi_{2}(z)=\frac{P^{\prime}(f) f^{\prime}(z)}{P(f)}-\frac{\Phi^{\prime}}{\Phi}$. If $z_{0}$ is a zero of $\Phi(z)$, zero of $P(f(z))$ or pole of $f(z+c)$, then $z_{0}$ is a simple pole of $\varphi_{2}(z)$; if $z_{0}$ is a pole of $P(f(z))$ but not a pole of $f(z+c)$, using the Laurent series, we can get that $\varphi_{2}(z)$ is analytic at $z_{0}$. Therefore, we conclude that

$$
\begin{aligned}
& N\left(r, \frac{P^{\prime}(f) f^{\prime}(z)}{P(f)}-\frac{\Phi^{\prime}}{\Phi}\right) \\
\leq & \bar{N}\left(r, \frac{1}{\Phi(z)}\right)+\bar{N}\left(r, \frac{1}{P(f)}\right)+\bar{N}(r, f(z+c))+S(r, f(z)) \\
= & \bar{N}\left(r, \frac{1}{\Phi(z)}\right)+\sum_{i=1}^{m} \bar{N}\left(r, \frac{1}{f(z)-a_{i}}\right)+\bar{N}(r, f(z))+S(r, f(z)),
\end{aligned}
$$

where $a_{1}, a_{2}, \ldots, a_{m}$ are the solutions of equation $P(z)=0$.

Combining (3.4), (3.5) and (3.6), we have that

$$
\begin{aligned}
& n T(r, f(z)) \\
\leq & 2 m\left(r, \frac{\Phi^{\prime}}{\Phi}\right)+m(r, f(z))+m\left(r, \frac{f^{\prime}(z+c)}{f(z+c)}\right)+\sum_{i=1}^{m} \bar{N}\left(r, \frac{1}{f(z)-a_{i}}\right)
\end{aligned}
$$




$$
+2 \bar{N}\left(r, \frac{1}{\Phi(z)}\right)+2 \bar{N}(r, f(z))+N(r, f(z))+S(r, f(z)) .
$$

From (3.2) and Lemma 2.2, we deduce that $T(r, \Phi(z))=O(T(r, f(z))$. Therefore, we get that

$$
m\left(r, \frac{f^{\prime}(z+c)}{f(z+c)}\right)=S(r, f(z)), m\left(r, \frac{\Phi^{\prime}}{\Phi}\right)=S(r, \Phi(z))=S(r, f(z)) .
$$

By (3.8) and the First Fundamental Theorem, we can simplify (3.7) to be

$$
(n-m) T(r, f(z)) \leq 3 T(r, f(z))+2 \bar{N}\left(r, \frac{1}{\Phi(z)}\right)+S(r, f(z)) .
$$

Because of $n-m>3$, we deduce that

$$
T(r, f(z)) \leq C \bar{N}\left(r, \frac{1}{P(f)+f(z+c)-s(z)}\right)+S(r, f(z)) .
$$

If $P(f)+f(z+c)-s(z)$ has no zero, then $T(r, f(z))=S(r, f(z))$, a contradiction.

When $f$ is transcendental, by the same reason, we conclude that $P(f)+$ $f(z+c)-s(z)$ has infinite many zeros.

This completes the proof of Theorem 1.1.

Proof of Theorem 1.2. We define $\Phi(z)=P(f(z))+f(q z)-s(z)$. The following process is almost literally the same as the proof of Theorem 1.1, with Lemmas 2.3 and 2.4 replacing Lemmas 2.1 and 2.5.

This completes the proof of Theorem 1.2.

Acknowledgment. The authors would like to express their hearty thanks to Professor Walter Bergweiler for his valuable advice. The first authors thank the Department of Mathematics, Kiel university of Germany, for its hospitality during the study period there. The authors are grateful to the reviews for their valuable comments which lead to the improvement of this paper.

\section{References}

[1] D. Barnett, R. G. Halburd, W. Morga, and R. J. Korhonen, Nevanlinna theory for the qq-difference operator and meromorphic solutions of qq-difference equations, Proc. Roy. Soc. Edinburgh Sect. A 137 (2007), 457-474.

[2] W. Bergweiler and J. K. Langley, Zeros of difference of meromorphic functions, Math. Proc. Cambridge Philos. Soc. 142 (2007), no. 1, 133-147.

[3] Z. X. Chen, On value distribution of difference polynomial of meromorphic functions, Abstr. Appl. Anal. 2011 (2011), 1-9.

[4] Y. M. Chiang and S. J. Feng, On the growth of logarithmic differences, difference quotients and logarithmic derivatives of meromorphic functions, Trans. Amer. Math. Soc. 361 (2009), no. 7, 3767-3791.

[5] On the Nevanlinna characteristic $f(z+\eta)$ and difference equations in the complex plane, Ramanujan J. 16 (2008), no. 1, 105-129.

[6] J. Grahl, Differential polynomial with dilations in the argument and normal families, Monatsh. Math. 162 (2011), no. 4, 429-452. 
[7] R. G. Halburd and R. J. Korhonen, Difference analogue of the lemma on the Logarithmic Derivative with application to difference equations, J. Math. Anal. Appl. 314 (2006), no. $2,477-487$.

[8] , Nevanlinna theory for the difference operator, Ann. Acad. Sci. Fenn. Math. 31 (2006), no. 2, 463-478.

[9] Finite-order meromorphic solutions and the discrete Painleve equations, Proc. Lond. Math. Soc. 94 (2007), no. 2, 443-474.

[10] W. K. Hayman, Picard values of meromorphic functions and their derivatives, Ann. of Math. 70 (1959), 9-42.

[11] _ Meromorphic Functions, Clarendon Press, Oxford, UK, 1964.

[12] J. Heittokangas, R. Korhonen, I. Laine, J. Rieppo, and K. Tohge, Complex difference equations of Malmquist type, Comput. Methods Funct. Theory 1 (2001), no. 1, 27-39.

[13] J. Heittokangas, R. Korhonen, I. Laine, J. Rieppo, and L. J. Zhang, Value sharing results for shifts of meromorphic function and sufficient conditions for periodicity, J. Math. Anal. Appl. 355 (2009), no. 1, 352-363.

[14] K. Ishizaki and N. Yanagihara, Wiman-Valiron method for difference equations, Nagoya Math. J. 175 (2004), 75-102.

[15] I. Laine, Nevanlinna Theory and Complex Differential Equation, Walter de Gruyter, Berlin-New York, 1993.

[16] I. Laine and C. C. Yang, Clunie theorems for difference q-difference polynomials, J. Lond. Math. Soc. 76 (2007), no. 3, 556-566.

[17] _ Value distribution of difference polynomials, Proc. Japan Acad. Ser. A Math. Sci. 83 (2007), no. 8, 148-151.

[18] K. Liu and I. Laine, A note on value distribution of difference polynomial, Bull. Aust. Math. Soci. 81 (2010), no. 3, 353-360.

[19] K. Liu and L. Z. Yang, Value distribution of the difference operator, Arch. Math. 92 (2009), no. 3, 270-278.

[20] A. I. Markushevich, Theory of Functions of a Complex Variable. Vol. 2, Englewood Cliffs, N. J. Prentice-Hall, 1965.

[21] J. M. Qi, J. Ding, and T. Y. Zhu, Some results about a special nonlinear difference equation and uniqueness of diffrence polynomial, J. Inequal. Appl. 2011 (2011), 50, $1-10$.

[22] X. G. Qi and K. Liu, Uniqueness and value distribution of differences of entire functions, J. Math. Anal. Appl. 379 (2011), no. 1, 180-187.

[23] C. C. Yang and H. X. Yi, Uniqueness Theory of Meromorphic Functions, Kluwer Academic Publishers, 2004.

[24] J. L. Zhang and R. Korhonen, On the Nevanlinna characteristic of $f(q z)$ and its applications, J. Math. Anal. Appl. 369 (2010), no. 2, 537-544.

JIE DING

School of Mathematics

Taiyuan University of Technology

Taiyuan, Shanxi, 030024, P. R. China

E-mail address: jieding1984@gmail.com

LiAN-ZHONG YANG

SCHOOL OF MATHEMATICS

SHANDONG UNIVERSITY

Jinan, Shandong, 250100, P. R. China

E-mail address: lzyang@sdu.edu.cn 